\title{
Direct and inverse cascades in the geodynamo
}

\author{
M. Reshetnyak ${ }^{1}$ and P. Hejda ${ }^{2}$ \\ ${ }^{1}$ Institute of the Physics of the Earth, Russian Acad. Sci, 123995 Moscow, Russia \\ ${ }^{2}$ Institute of Geophysics, Academy of Sciences, 14131 Prague, Czech Republic \\ Received: 4 April 2008 - Revised: 25 June 2008 - Accepted: 29 September 2008 - Published: 24 November 2008
}

\begin{abstract}
The rapid rotation of planets causes cyclonic thermal turbulence in their cores which may generate the largescale magnetic fields observed outside the planets. We investigate numerically a model based on the geodynamo equations in simplified geometry, which enables us to reproduce the main features of small-scale geostrophic flows in physical and wave vector spaces. We find fluxes of kinetic and magnetic energy as a function of the wave number and demonstrate the co-existence of forward and inverse cascades. We also explain the mechanism of magnetic field saturation at the end of the kinematic dynamo regime.
\end{abstract}

\section{Introduction}

Many astrophysical objects such as galaxies, stars, the Earth, and some planets have large-scale magnetic fields that are believed to be generated by a common universal mechanism - the conversion of kinetic energy into magnetic energy in a turbulent rotating shell. The details, however, and thus the nature of the resulting field, differ greatly. The challenge for the dynamo theory, see, e.g., Hollerbach and Rüdiger (2004), is to provide a model that can explain the visible features of the field with realistic assumptions of the model parameters. Calculations for an entire planet are done using either spectral models (Kono and Roberts, 2002) finite-volume methods (Hejda and Reshetnyak, 2004; Harden and Hansen, 2005) or finite differences (Kageyama and Sato, 1997) and have demonstrated beyond reasonable doubt that the turbulent 3-D convection of the conductive fluid can generate a large-scale magnetic field similar to the one associated with small random fluctuations. However, both of these methods cannot cover the enormous span of scales required for a realistic parameter set. Even for the geodynamo (which is quite a modest case on the astrophysical scale) the hydro-

Correspondence to: M. Reshetnyak (m.reshetnyak@gmail.com) dynamic Reynolds number estimated on the west-drift velocity is $\operatorname{Re} \sim 10^{9}$. In addition, planets are rapidly rotating bodies. Thus, the time scale of the large-scale convection in the Earth's core is $\sim 10^{3}$ years, during which the planet itself makes $\sim 10^{6}$ revolutions (in other words, the Rossby number Ro $\sim 10^{-6}$ ). As a result, there is an additional spatial scale $\sim \mathrm{E}^{-1 / 3} L$, where $L$ is a large scale, $\mathrm{E} \sim 10^{-15}$ is the Ekman number (Chandrasekhar, 1961; Busse, 1970), associated with the cyclonic structures elongated along the axis of rotation, which is much larger than Kolmogorov's dissipation scale $l_{d} \sim \mathrm{Re}^{-4 / 3} L$, but, however, is still too small to be resolved in the numerical simulations with the present resolution $l \sim\left(10^{-3} \div 10^{-2}\right) L$.

The presence of rapid rotation leads not only to a change from the spatially uniform, isotropic, Kolmogorov-like solution to the quasi-geostrophic (magnetostrophic) form, but to rather more fundamental consequences. The rapid rotation leads to the degeneration of the third dimension (along the axis of rotation) and can cause an inverse cascade in the system. Inverse cascades are a well-known phenomenon in two-dimensional turbulence and are a good example of selforganization when the large-scale structures are fed by smallscale turbulence (Frisch et al., 1975; Kraichnan and Montgomery, 1980; Tabeling, 2002), see also review of the recent results in Alexakis (2007); Verma (2004). As the quasigeostrophy is an intermediate state between two-dimensional and three-dimensional flows, the quasi-geostrophic turbulence may exhibit simultaneously features similar to both the extreme cases: 2-D and 3-D ${ }^{1}$. Below we consider the behavior of the energy fluxes in the wave space for regimes based on the Boussinesque thermal convection. For simplicity we consider the Cartesian geometry, which is simpler for the modeling of rapidly rotating dynamo systems and was used in many geodynamo research projects (Roberts, 1999; Jones and Roberts, 2000; Buffett, 2003).

\footnotetext{
${ }^{1}$ As the magnetic field generation is a three-dimensional process, see, e.g., Zeldovich et al. (1983), existence of the third dimension in the full dynamo problem is crucial.
} 


\section{Dynamo equations}

\subsection{Equations in physical space}

The geodynamo equations for an incompressible fluid $(\nabla \cdot V=0)$ in a layer of the height $\mathrm{L}$ rotating with angular velocity $\Omega$ in the Cartesian system of coordinates $(x, y, z)$ in its traditional dimensionless form can be expressed as follows:

$$
\begin{aligned}
& \frac{\partial \boldsymbol{B}}{\partial t}=\nabla \times(\boldsymbol{V} \times \boldsymbol{B})+\mathrm{q}^{-1} \Delta \boldsymbol{B} \\
& \operatorname{EPr}^{-1}\left[\frac{\partial \boldsymbol{V}}{\partial t}+(\boldsymbol{V} \cdot \nabla) \boldsymbol{V}\right]=-\nabla P-\mathbf{1}_{z} \times \boldsymbol{V}+ \\
& \operatorname{Ra} T z \mathbf{1}_{z}+(\nabla \times \boldsymbol{B}) \times \boldsymbol{B}+\mathrm{E} \Delta \boldsymbol{V} \\
& \frac{\partial T}{\partial t}+(\boldsymbol{V} \cdot \nabla)\left(T+T_{0}\right)=\Delta T .
\end{aligned}
$$

Velocity $\boldsymbol{V}$, magnetic field $\boldsymbol{B}$, pressure $P$ and the typical diffusion time $t$ are measured in units of $\kappa / \mathrm{L}, \sqrt{2 \Omega \kappa \mu \rho}$; $\rho \kappa^{2} / \mathrm{L}^{2}$ and $\mathrm{L}^{2} / \kappa$, respectively, where $\kappa$ is the thermal diffusivity, $\rho$ is the density, $\mu$ the permeability, $\operatorname{Pr}=\frac{\kappa}{v}$ is the Prandtl number, $\mathrm{E}=\frac{v}{2 \Omega \mathrm{L}^{2}}$ is the Ekman number, $v$ is the kinematic viscosity, $\eta$ is the magnetic diffusivity, and $\mathrm{q}=\kappa / \eta$ is the Roberts number. $\mathrm{Ra}=\frac{\alpha \mathrm{g}_{0} \delta \mathrm{TL}}{2 \Omega \kappa}$ is the modified Rayleigh number, $\alpha$ is the coefficient of volume expansion, $\delta T$ is the unit of temperature, for more details see (Jones, 2000) , $g_{0}$ is the gravitational acceleration, and $T_{0}=1-z$ is the heating from below. The problem is closed with periodical boundary conditions in the $(x, y)$ plane. In the $z$-direction, we use simplified conditions (Cattaneo et al., 2003): $T=0, V_{z}=\frac{\partial V_{x}}{\partial z}=\frac{\partial V_{y}}{\partial z}=0, B_{x}=B_{y}=\frac{\partial B_{z}}{\partial z}=0$ at $z=0,1$.

\subsection{Equations in wave space}

To solve problem Eq. (1) we apply the pseudo-spectral approach (Orszag, 1971) frequently used in geodynamo simulations (Jones and Roberts, 2000; Buffett, 2003). The equations are solved in the wave space. To calculate the nonlinear terms one needs to make the inverse Fourier transform, then calculate the product in physical space, make the Fourier transform of the product, and finally calculate the derivatives in wave space. After eliminating the pressure using the divergence-free condition $\boldsymbol{k} \cdot \boldsymbol{V}=0, \boldsymbol{k} \cdot \boldsymbol{B}=0$, we arrive at:

$$
\begin{aligned}
& {\left[\frac{\partial \boldsymbol{B}}{\partial t}+\mathrm{q}^{-1} k^{2} \boldsymbol{B}\right]_{k}=[\nabla \times(\boldsymbol{V} \times \boldsymbol{B})]_{\boldsymbol{k}}} \\
& \mathrm{E}\left[\operatorname{Pr}^{-1} \frac{\partial \boldsymbol{V}}{\partial t}+k^{2} \boldsymbol{V}\right]_{\boldsymbol{k}}=\boldsymbol{k} \mathcal{P}_{\boldsymbol{k}}+\boldsymbol{F}_{\boldsymbol{k}} \\
& {\left[\frac{\partial T}{\partial t}+k^{2} T\right]_{k}=-\left[(\boldsymbol{V} \cdot \nabla) T+V_{r}\right]_{\boldsymbol{k}}}
\end{aligned}
$$

with

$$
\begin{aligned}
& \mathcal{P}_{\boldsymbol{k}}=-\frac{\boldsymbol{k} \cdot \boldsymbol{F}_{\boldsymbol{k}}}{k^{2}}, \quad k^{2}=k_{\beta} k_{\beta}, \quad \beta=1 \ldots 3 \\
& \boldsymbol{F}_{\boldsymbol{k}}= {\left[\operatorname{Pr}^{-1} \boldsymbol{V} \times(\nabla \times \boldsymbol{V})+\operatorname{Ra} T \mathbf{1}_{z}-\right.} \\
&\left.\mathbf{1}_{z} \times \boldsymbol{V}+(\boldsymbol{B} \cdot \nabla) \boldsymbol{B}\right]_{k} .
\end{aligned}
$$

For integration in time we use the explicit Adams-Bashforth (AB2) scheme for non-linear terms. The linear terms are treated using the Crank-Nicolson $(\mathrm{CN})$ scheme. To resolve the diffusion terms we use the well-known trick which helps to increase the time step significantly. Consider equation

$\frac{\partial A}{\partial t}+k^{2} A=U$.

Alter it to read

$\frac{\partial A e^{k^{2} \gamma t}}{\partial t}=U e^{k^{2} \gamma t}$

and then apply the $\mathrm{CN}$ scheme.

The most time-consuming part of our MPI code are the Fast Fourier transforms. To make our code more efficient we use various modifications of known FFT algorithms, which take into account special kinds of symmetry of the fields. The optimal number of processors for the grids $128^{3}$ is $n \sim 50$. The scalability tests demonstrated even the presence of superacceleration if the number of processors $<n$.

\section{Basic properties of the fields}

We consider simulations without rotation similar to Meneguzzi and Pouquet (1989) and with rotation for two regimes with different amplitudes of the heat sources:

NR: Regime without rotation (the Coriolis term is dropped $)^{2}$. $\mathrm{Ra}=6 \cdot 10^{6}, \operatorname{Pr}=1, \mathrm{E}=1, \mathrm{q}=10, \operatorname{Re} \sim 2.5 \cdot 10^{3}$.

$\mathrm{R} 1$ : Regime with rotation, $\mathrm{Ra}=1.3 \cdot 10^{3}, \operatorname{Pr}=1, \mathrm{E}=2 \cdot 10^{-6}$, $\mathrm{q}=10, \operatorname{Re} \sim 1.6 \cdot 10^{3}$.

$\mathrm{R} 2$ : Regime with rotation, $\mathrm{Ra}=2.1 \cdot 10^{3}, \operatorname{Pr}=1, \mathrm{E}=2 \cdot 10^{-6}$, $\mathrm{q}=10, \operatorname{Re} \sim 3 \cdot 10^{3}$.

The first (NR) regime is close to the typical Kolmogorov convection; for more details see Meneguzzi and Pouquet (1989). Inclusion of rotation (regime R1, Figs. 1-5) leads to the transform of the isotropic convective structures to the cyclonic state with the horizontal scale $\sim \mathrm{E}^{1 / 3}\left(k_{c} \sim \mathrm{E}^{-1 / 3}\right)$ (Chandrasekhar, 1961). Inclusion of the magnetic field (the full dynamo regime with magnetic energy comparable with

\footnotetext{
${ }^{2}$ As there is no rotation, $\mathrm{Ra}$ and $\mathrm{E}$ do not retain their physical meaning defined in Sect. 2.1. More details on parametrization of non-rotational magnetoconvection can be found in Meneguzzi and Pouquet (1989).
} 

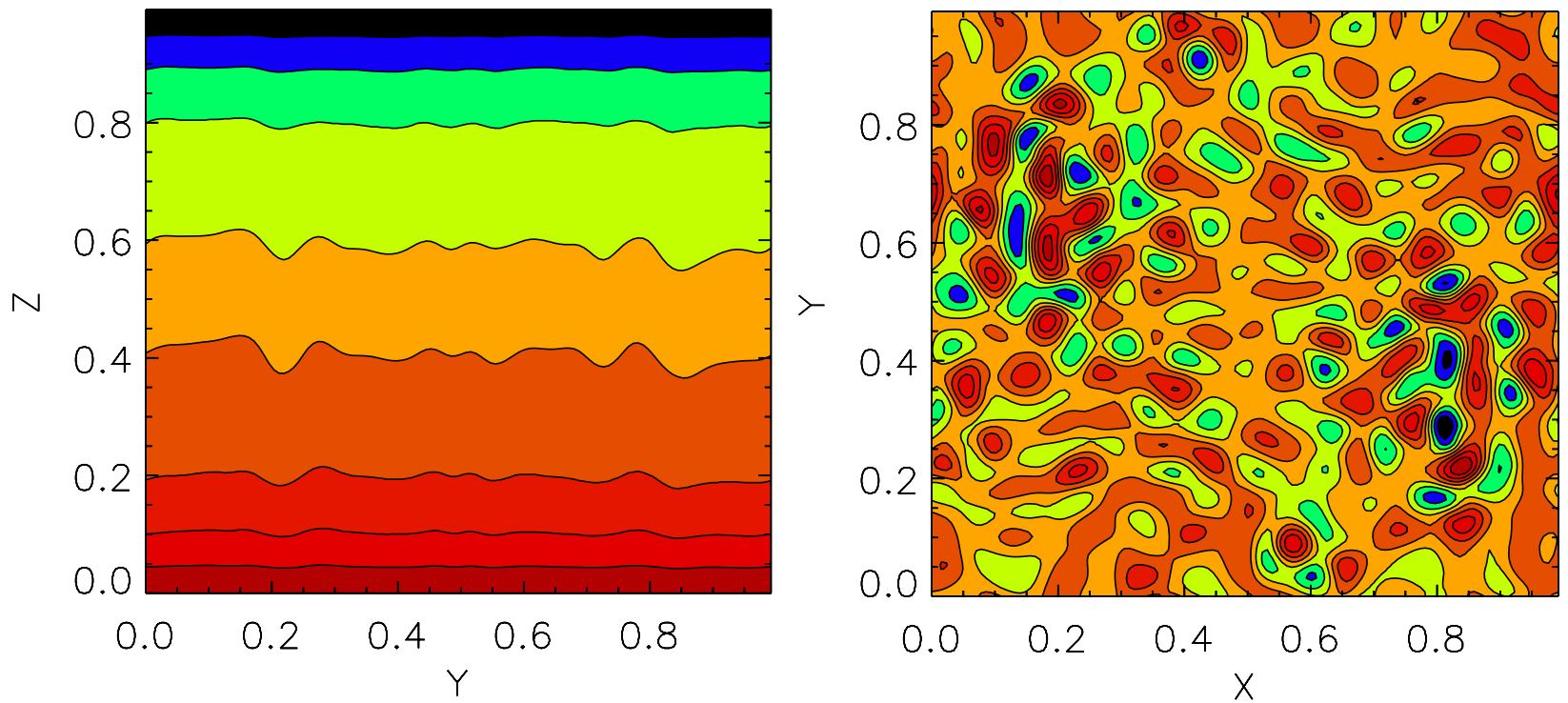

Fig. 1. Regime R1. Sections of temperature distribution. All sections correspond to the middle of the cube. The field ranges are $(0,1)-$ left, $(0.46,0.54)$ - right.
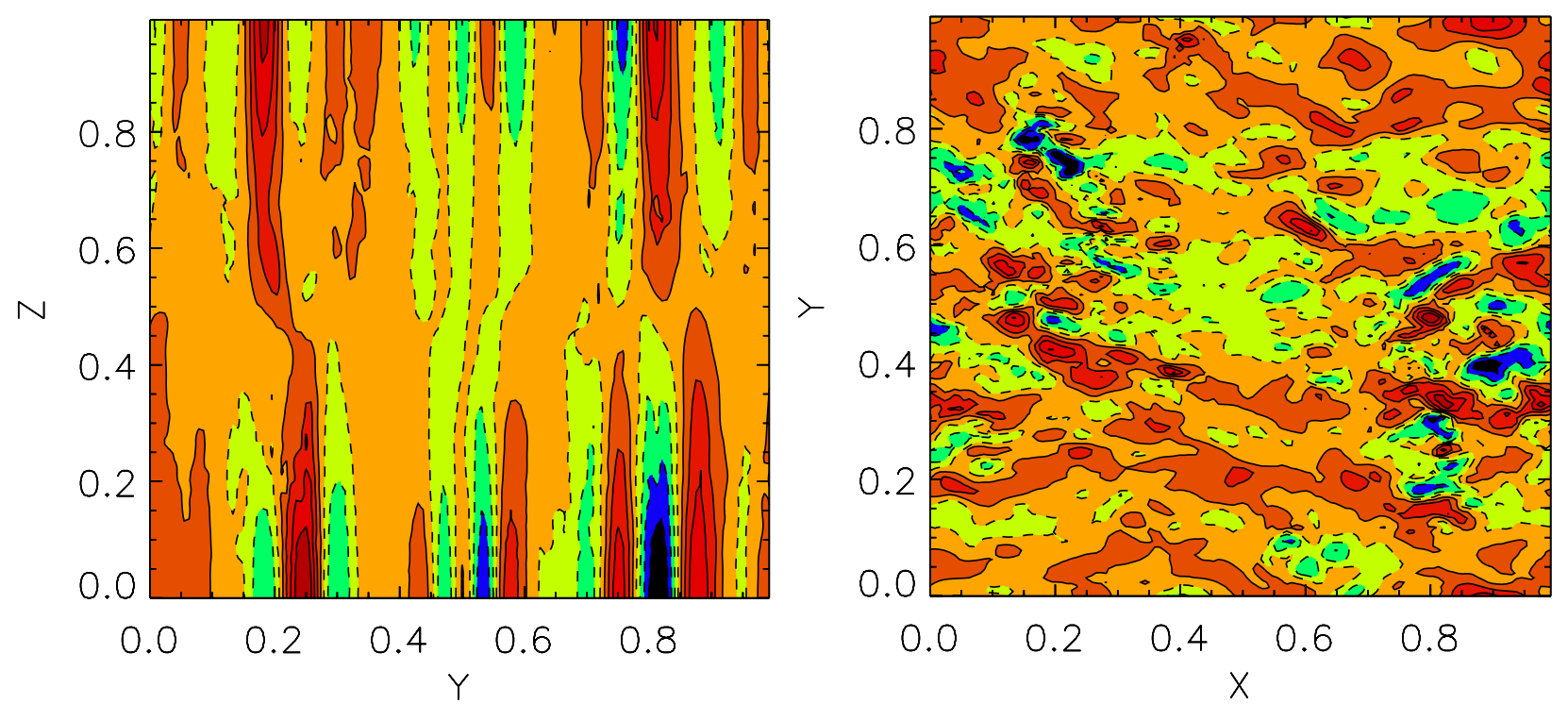

Fig. 2. Distribution of the $V_{x}$-component of the velocity field with ranges $(-248,253),(-143,144)$.

the kinetic energy in order of magnitude) does not change the structure of the convective patterns very much (Jones, 2000). At the same time, the spectra of magnetic energy are quite different and have no well-pronounced maximum at $k_{c}$.

The increase of the Rayleigh number leads to the decrease of the relative role of rotation and should decrease the peak of the kinetic spectra energy, which is in accordance with the spectra for regime R2, Fig. 5. In principle, a further increase of Ra should lead to the original Kolmogorov state, similar to NR with the spectrum law $\sim k^{-5 / 3}$, Fig. 5. However, we emphasize that the information on the spectra is not enough to judge, if the role of rotation is negligible or not, and additional analysis is needed. The argument is as follows: rotation leads to the degeneration of the third dimension (along the z-axis) (Batchelor, 1953). On the other hand, in isotropic two-dimensional systems, the spectrum of the kinetic energy also has a $-5 / 3$-slope, but the direction of the energy transfer at the large scales is inverse (there is also a direct cascade 

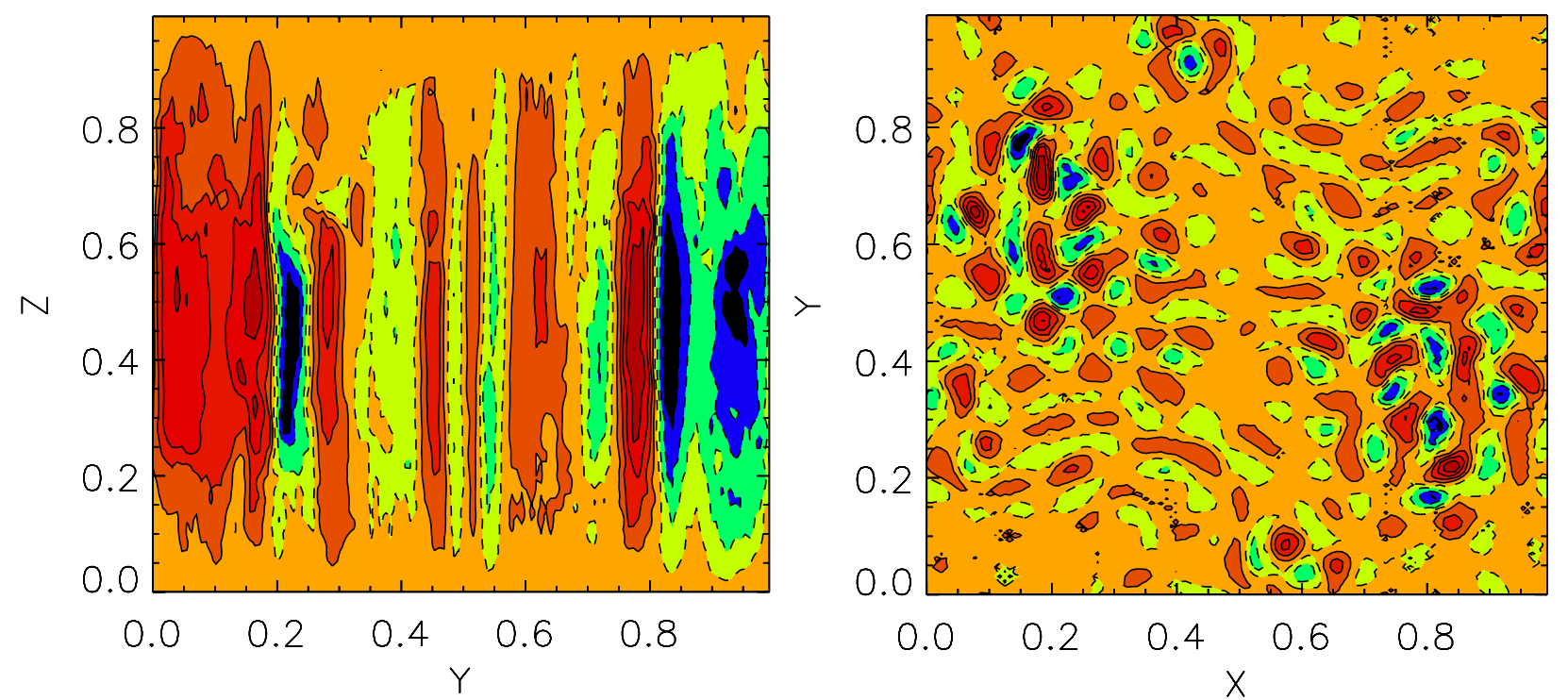

Fig. 3. Distribution of the $V_{z}$-component of the velocity field with ranges $(-675,701),(-153,157)$
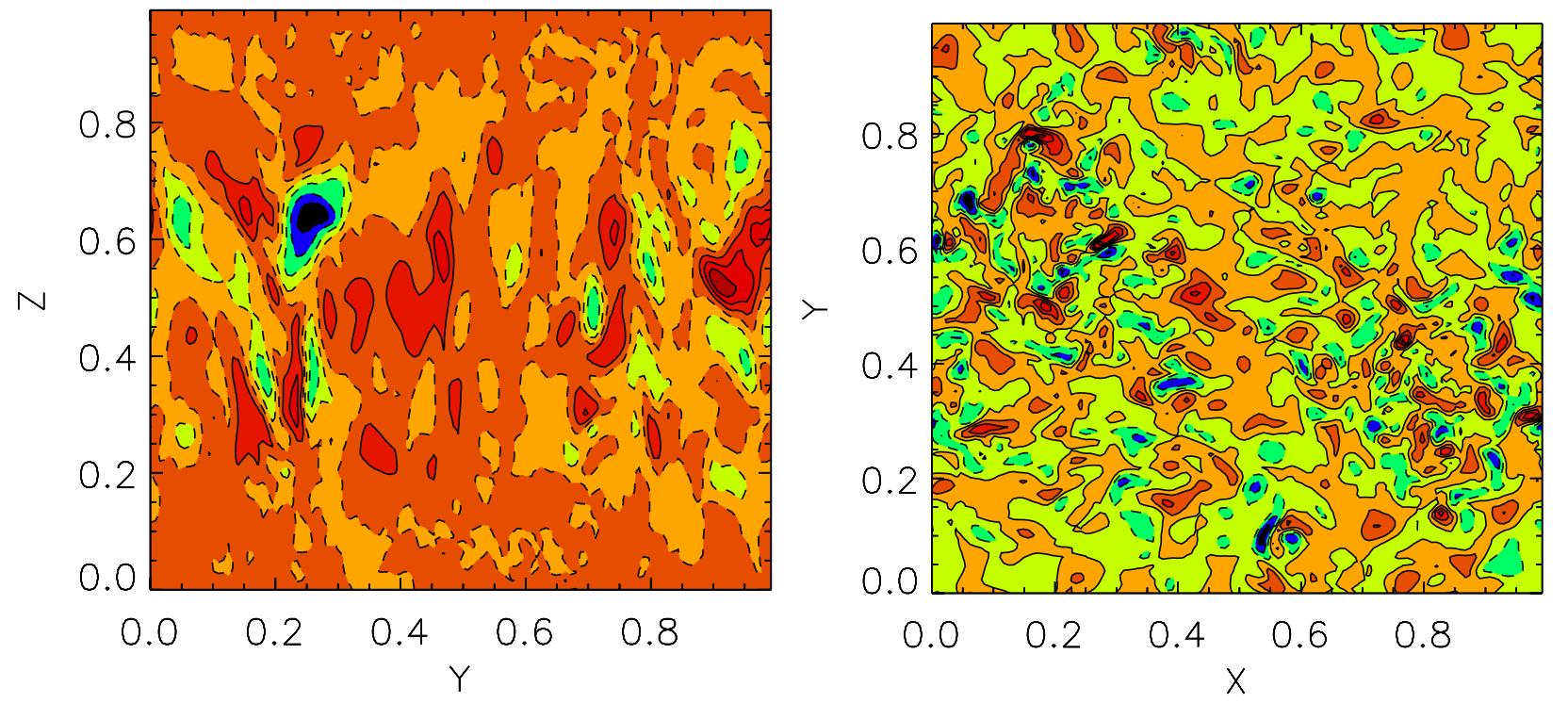

Fig. 4. Distribution of the $B_{z}$-component of the magnetic field with ranges $(-1.44,1.14),(-1.88,2.37)$

of the enstrophy at the small scales with a -3-slope). As the quasi-geostrophic turbulence inherits the properties of both systems, 2-D and 3-D, (Hossain, 1994; Constantin, 2002), we will consider the behavior of the energy fluxes in wave space more carefully.

\section{Energy fluxes}

To analyze the energy transfer in the wave space, we follow Frisch (1995). Let us decompose the physical field $f$ into a sum of low-frequency and high-frequency counterparts: $f(\boldsymbol{r})=f^{<}(\boldsymbol{r})+f^{>}(\boldsymbol{r})$, where

$$
f^{<}(\boldsymbol{r})=\sum_{|k| \leq K} \widehat{f_{k}} e^{i \boldsymbol{k} \boldsymbol{r}}, \quad f^{>}(\boldsymbol{r})=\sum_{|k|>K} \widehat{f_{k}} e^{i \boldsymbol{k} \boldsymbol{r}} .
$$



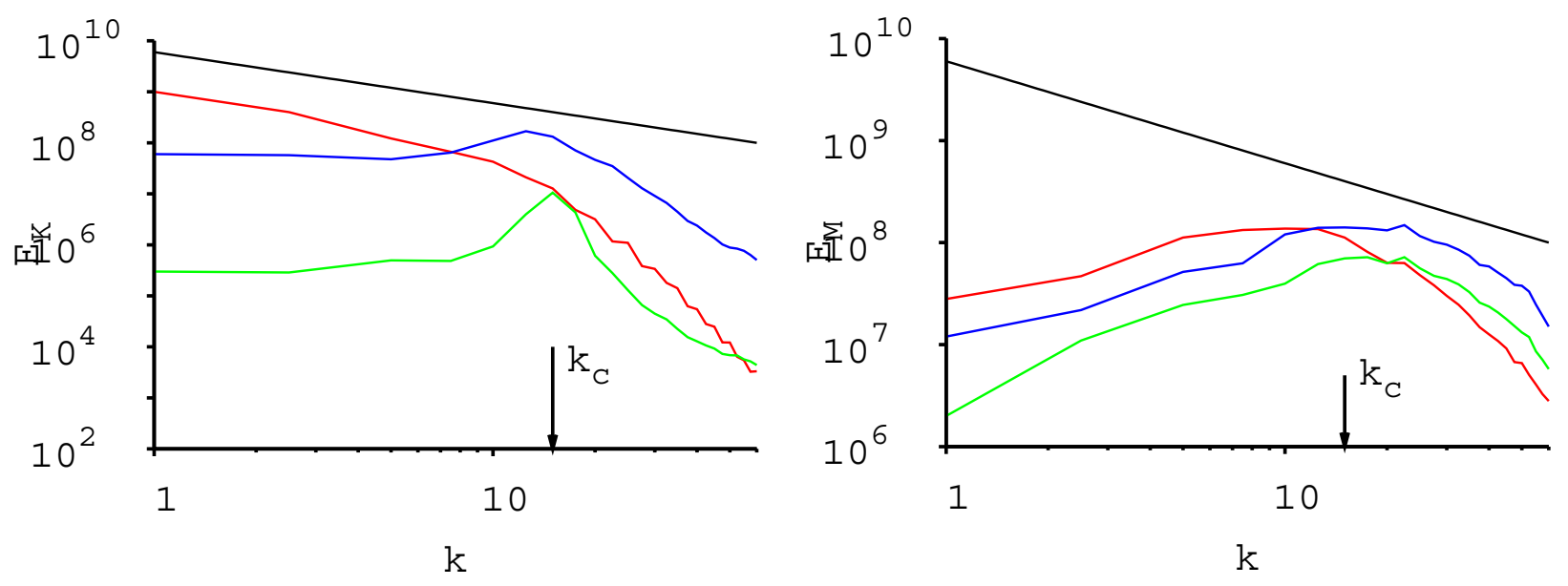

Fig. 5. On the left is the spectrum of the kinetic energy for NR (red), R1 (green), R2 (blue). On the right is the spectrum of the magnetic energy. The straight line corresponds to Kolmogorov's spectrum $\sim k^{-5 / 3}$.

For any periodical functions $f$ and $g$ one has the relation (Frisch, 1995):

$$
\begin{gathered}
<\frac{\partial f}{\partial x}>=0, \quad<\frac{\partial g}{\partial x}>=0, \\
<g \frac{\partial f}{\partial x}>=-<f \frac{\partial g}{\partial x}>, \quad<f^{>} g^{<}>=0,
\end{gathered}
$$

where

$$
<f(\boldsymbol{r})>=\mathcal{V}^{-1} \int_{\mathcal{V}} f(\boldsymbol{r}) d \boldsymbol{r}^{3}
$$

stands for averaging of $f$ over volume $\mathcal{V}$. Multiplying the Navier-Stokes equation by $\boldsymbol{V}^{<}$and the induction equation by $\boldsymbol{B}^{<}$leads to the equations of the integral fluxes of the kinetic $E_{K}=V^{2} / 2$ and magnetic $E_{M}=B^{2} / 2$ energies from $k \geq K$ to $k<K$ :

$$
\begin{aligned}
& \Pi_{K}(K)=<(\boldsymbol{V} \times \operatorname{rot} \boldsymbol{V}) \cdot \boldsymbol{V}^{<}>, \\
& \Pi_{M}(K)=<\operatorname{rot}(\boldsymbol{V} \times \boldsymbol{B}) \cdot \boldsymbol{B}^{<}>
\end{aligned}
$$

and for the flux of the Lorentz work:

$$
\Pi_{L}(K)=<(\operatorname{rot} \boldsymbol{B} \times \boldsymbol{B}) \cdot \boldsymbol{V}^{<}>.
$$

Here subscripts $K, M$ and $L$ correspond to kinetic, magnetic and Lorentz fluxes. Introducing

$T_{K}(k)=-\frac{\partial \Pi_{K}(k)}{\partial k}$,

where we changed $K$ to $k$, leads to the obvious relation for $E_{K}$ in $k$-space:

$$
\frac{\partial E_{K}(k)}{\partial t}=T_{K}(k)+F(k)+D(k),
$$

where $k=|\boldsymbol{k}|, T_{K}(k)$ is the kinetic energy flux from harmonics with different $k, F(k)$ is the work of external forces and $D(k)=-k^{2} E_{K}(k)$ is a dissipation. The accurate form of $T$ reads:

$$
\begin{aligned}
& T_{K}=-\frac{\partial \Pi_{K}}{\partial k}, \quad \int_{k=0}^{\infty} T_{K}(k) d k=0, \\
& T_{M}=\frac{\partial \Pi_{M}}{\partial k}, \quad T_{L}=\frac{\partial \Pi_{L}}{\partial k} .
\end{aligned}
$$

We introduce advective flux $T_{N}=T_{M}+T_{L}$ with zero average:

$$
\int_{k=0}^{\infty} T_{N}(k) d k=0 .
$$

Figure 6 shows the fluxes of kinetic $T_{K}$ and magnetic $T_{M}$ energies for the regimes mentioned above. Regime NR for $T_{K}$ demonstrates the well-known behavior for the direct Kolmogorov's cascade in 3-D. For large scales $T_{K}<0$, these scales are donors and provide energy to the system. On the other hand, the harmonics with the large $k$ absorb energy. The two-dimensional turbulence exhibits mirrorsymmetrical behavior relative to the axis of the absciss (Kraichnan and Montgomery, 1980). In this case the energy cascade is inverse.

Rotation essentially changes the behavior of the fluxes of kinetic energy. The leading order wave number is $k_{c}$. For $k>k_{c}$ we also observe the direct cascade of energy $T_{K}>0$. The maximum of $T_{K}$ is shifted relative to the maximum of the energy to large $k$; the more Re, the stronger the shift. For $k<k_{c}$, the behavior is more complex: for small $k$, the inverse cascade of kinetic energy takes place, $T_{K}>0$. On the other hand, for the larger region of $k\left(0 \ldots k_{c}\right)$ we still have the direct cascade $T_{K}<0$. The increase of Re leads to the narrowing of the region with the inverse cascade and to the increase 

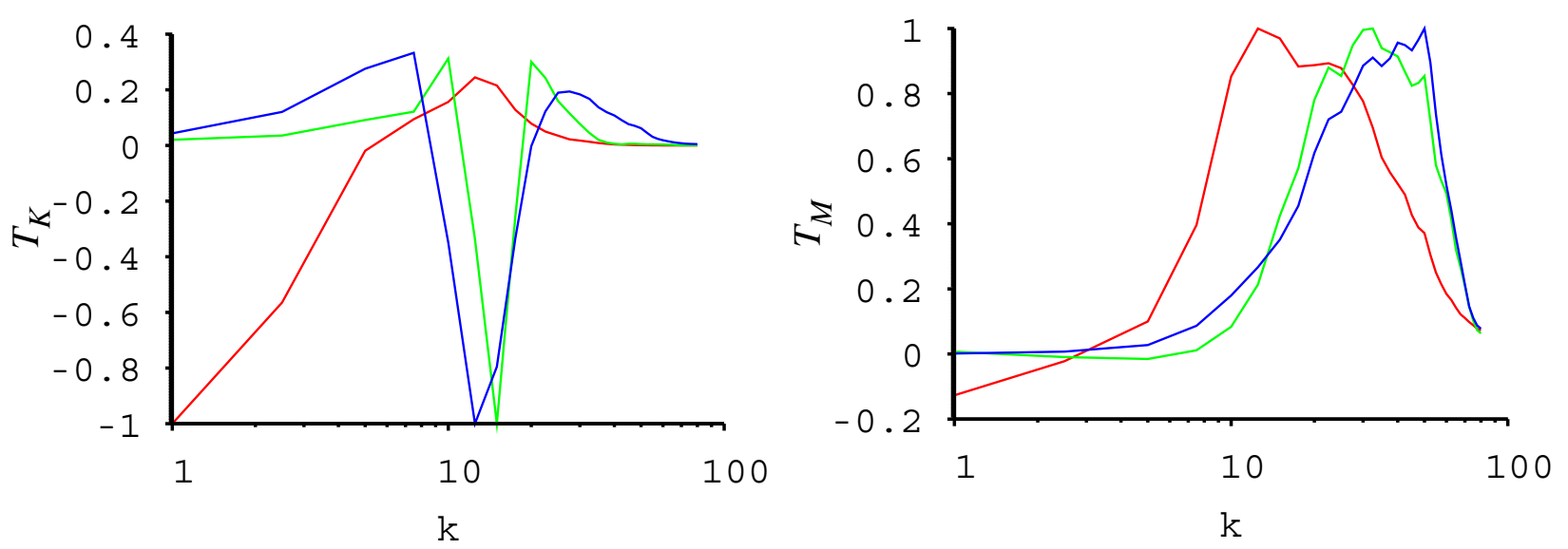

Fig. 6. Normalized fluxes of kinetic $T_{K}$ (on the left) and magnetic $T_{M}$ (on the right) energies in wave space: NR (red), R1 (green), R2 (blue).
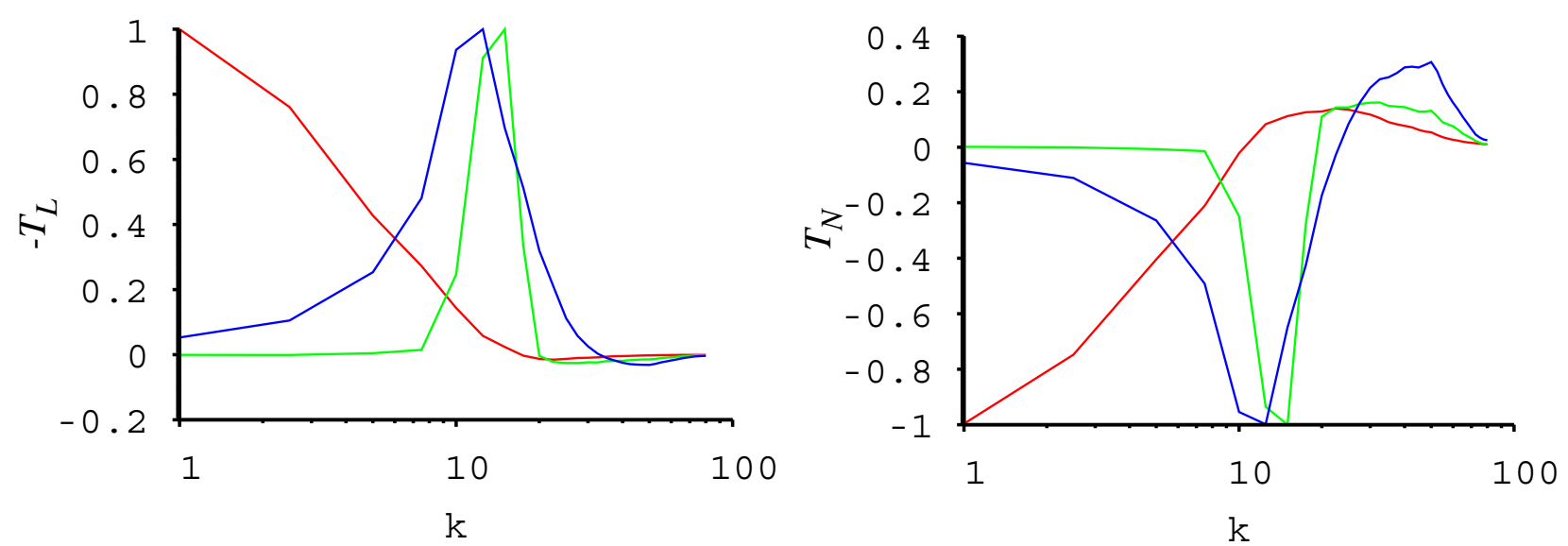

Fig. 7. Normalized fluxes of the generation term $-T_{L}$ (on the left) and advective $\mathrm{c} T_{N}$ (on the right) terms in wave space: $\mathrm{NR}$ (red), R1 (green), R2 (blue).

of the inverse flux. One may suggest that the change of the sign of flux $T_{K}$ at $k<k_{c}$ is connected with the appearance of the non-local energy transfer: so that the energy to the largescales $\boldsymbol{k}_{1}$ comes from modes $\left|\boldsymbol{k}_{2}\right| \sim\left|\boldsymbol{k}_{3}\right| \gg\left|\boldsymbol{k}_{1}\right|, \boldsymbol{k}_{1}=\boldsymbol{k}_{2}+\boldsymbol{k}_{3}$ (Waleffe, 1992). In the absence of the magnetic field the maximum of $T_{K}(k=1)$ appears. Hence, in the case of rotation, two cascades of kinetic energy (direct and inverse) take place simultaneously.

Now we consider the magnetic part. In contrast to $T_{K}$, $T_{M}$ includes not only the advective term, but also the generative term. This leads to integral $T_{M}$ being positive over all $k$. Moreover, $T_{M}$ is positive for any $k$. The position of the maximum of $T_{M}$ is close to those in the spectra of $E_{M}, T_{K}$.

It is evident that, for planetary cores, the distance between the maxima in fluxes $T_{M}$ for NR and R1, R2 can be quite large, however, not as large as $k_{c}$. This statement concerns the condition on magnetic field generation, which holds when the local magnetic Reynolds number $r_{m}>1$ on the scale
$1 / k: \mathrm{r}_{\mathrm{m}}=\frac{v_{k}}{k \eta}>1$ and that for the planets $\eta \gg \nu$. At the same moment, the fluxes with small $k$ are small, i.e., the system is in a state of statistical equilibrium: dissipation on small scales is negligible.

Now we examine the origin of the magnetic energy on scale $1 / k$ : Is it connected with the energy transfer from the other scales or is it a product of real generation on this scale?

Figure 7 demonstrates the fluxes of $-T_{L}$ concerned with magnetic field generation. The maximum of the generation term without rotation is on a large scale, while for the rotating system it is at $\sim 1 / k_{c}$. Interestingly, for the rotating system there is a region $-T_{L}<0$ for large $k$, where the magnetic field reinforces convection. For regime NR, $-T_{L}$ drops quickly because of the kinetic energy decrease (Fig. 5). As a result we have: for the rotating system, the magnetic field is produced by the cyclones, while the large-scale dynamo operates for the non-rotating system. 
Now we estimate the role of the advective term $T_{N}$ separately. For the non-rotating regime, $T_{N}$ and $T_{K}$ are similar: the direct cascade takes place. For the rotating system, region $k \sim k_{c}$ is a source of energy. In contrast to $T_{K}, T_{N}$ has no positive regions at small $k$, i.e. the inverse cascade of the magnetic energy, related to the advective term in this region is absent. We draw attention to the amplitudes of fluxes $T_{M}$, $-T_{L}, T_{N}$ : for all three cases it holds that $\frac{\left|T_{M}\right|}{\left|T_{L}\right|} \sim 10^{-1}$, i.e., there exist two fluxes of magnetic energy in wave space with opposite directions. The first flux is related to the traditional energy transfer over the spectrum (advective term) and with the flux of the Lorentz work. The regions of maximal magnetic field generation coincide with the regions of the most effective magnetic energy transfer $T_{N}$ (from small $k$ to large $k$ ). This balance leads to an equipartional state, when dissipation takes place at large $k$.

Note that the full magnetic flux $T_{M}$ (Fig. 6) is localized at $k \gg 1$. For the non-rotating system, this is because the mean helicity and $\alpha$-effect are zero (Zeldovich et al., 1983). Thus the inverse cascade of the magnetic energy to small $k$ is absent.

For the rotating system there is a balance of the energy injection due to the Lorentz force and its advection to the large $k$. This effect reduces the $\alpha$-effect.

\section{Conclusions}

The magnetic fields of the planets are the main sources of information on the processes in their liquid cores on the time scales of $10^{2}-10^{3}$ years or longer. While the poloidal part of the magnetic field can be observed on the planet's surface, the largest component of field (toroidal) as well as the kinetic energy distrubution over the scales is absolutely invisible for the observer outside the core. Moreover, due to the finite conductivity of the mantle, even the poloidal part of the magnetic field is cut off at $k \ll k_{c}$. In other words, the part of the spectrum observable on the planets' surface is only small (not even the largest) of the whole spectrum of the field. That is why the importance of the numerical simulation can hardly be overestimated. Here we have shown that, in considering the quasigeostrophic state, both the cascades (direct and inverse) exist simulataneously. This is a challenge for the turbulent models of the geodynamo. The other interesting point is the nearly balance of the magnetic energy generation and the magnetic energy advection, which makes the net magnetic energy flux very small. Following our modelling the major part (up to $90 \%$ of the all generated magnetic energy) is advected to the large $k$. Then we could expect that the exponential growth of the magnetic energy in the kinematic regime $\left(T_{L} / T_{N} \sim k_{B} / k_{V} \gg 1\right.$, where $k_{B}, k_{V}$ are the integral wave numbers of the magnetic and velocity fields) stops and the system comes to the state, when $k_{B} \sim k_{V}$ and $T_{L}$ is substantial.
Acknowledgements. The research was supported by Grant No. IAA300120704 of the Grant Agency of the Academy of Sciences of the Czech Republic.

Edited by: V. Shrira

Reviewed by: two anonymous referees

\section{References}

Alexakis, A., Mininni, P. D., and Pouquet, A.: Turbulent cascades, transefer, and scale interactions in magentohydrodynamics, New J. Phys., 9, 1-20, 2007.

Batchelor, G. K.: The theory of homogeneous turbulence, Cambridge University Press, Cambridge, 1953.

Buffett, B.: A comparison of subgrid-scale models for large-eddy simulations of convection in the Earth's core, Geophys. J. Int., 153, 753-765, 2003.

Busse, F. H.: Thermal instabilities in rapidly rotating systems, J. Fluid Mech., 44, 441-460 , 1970.

Cattaneo, F., Emonet, T., and Weis, N.: On the interaction between convection and magnetic fields, Astrophys. J., 588, 1183-1198, 2003.

Chandrasekhar, S.: Hydrodynamic and hydromagnetic stability, Dover Publications. Inc., NY, 1961.

Constantin, P.: Energy spectrum of quasigeostrophic turbulence, Phys. Rev. Lett., 89(18), 184 501-184 504, 2002.

Frisch, U., Pouquet, A., Léorat, J., and Mazure, A..: Possibility of an inverse cascade of a magnetic helicity in magnetohydrodynamic turbulence, J. Fluid Mech., 68, part 4, 769-778, 1975.

Frisch, U.: Turbulence: the legacy of A. N. Kolmogorov, Cambridge University Press, Cambridge, 1995.

Harden, H. and Hansen, U.: A finite-volume solution method for thermal convection and dynamo problems in spherical shells, Geophys. J. Int., 161, 522-532, 2005.

Hejda, P. and Reshetnyak, M.: Control volume method for the thermal convection problem in a rotating spherical shell: test on the benchmark solution, Stud. Geophys. Geod., 48, 741-746, 2004.

Hollerbach, R. and Rüdiger, R.: The Magnetic Universe, WileyVCH Verlag GmbH \& Co. KGaA, Weinheim, 2004.

Hossain, M.: Reduction of the dimensionality of turbulence due to a strong rotation, Phys. Fluids. 6(4), 1077-1080, 1994.

Jones, C. A.: Convection-driven geodynamo models, Phil. Trans. R. Soc. London, 358, 873-897, 2000.

Jones, C. A. and Roberts, P. H.: Convection driven dynamos in a rotating plane layer, J. Fluid Mech., 404, 311-343, 2000.

Kageyama, A. and Sato, T.: Velocity and magnetic field structures in a magnetohydrodynamic dynamo, Phys. Plasma, 4(5), 1569$1575,1997$.

Kono, M. and Roberts, P.: Recent geodynamo simulations and observations of the geomagnetic field, Rev. Geophys., 40(10), B1B41, 2002.

Kraichnan, R. H. and Montgomery, D.: Two-dimensional turbulence, Rep. Prog. Phys., 43, 547-619, 1980.

Meneguzzi, M. and Pouquet, A.: Turbulent dynamos driven by convection, J. Fluid Mech., 205, 297-318, 1989.

Orszag, S. A.: Numerical simulation of incompressible flows within simple boundaries, I. Galerkin (spectral) representations, Stud. Appl. Math. L., 4, 293-327, 1971. 
Matsushima, M., Nakajima, T., and Roberts, P.: The anisotropy of local turbulence in the Earth's core, Earth Planets Space, 51, 277-286, 1999.

Tabeling, P.: Two-dimensional turbulence: a physicist approach. Phys. Reports., 362, 1-62, 2002.
Verma, M.: Statistical theory of magnetohydrodynamic turbulence: recent results, Phys. Reports, 401, 229-380, 2004.

Waleffe, F.: The nature of triad interactions in homogeneous turbulence, Phys. Fluids., A4(2), 350-363, 1992.

Zeldovich, Y. B., Ruzmaikin, A. A., and Sokoloff, D.D.: Magnetic fields in astrophysics, Gordon and Breach, NY, 1983. 